\title{
Class-Specific Synthesized Dictionary Model for Zero-Shot Learning
}

\author{
Zhong $\mathrm{Ji}^{\mathrm{a}, *}$, Junyue Wang ${ }^{\mathrm{a}}$, Yunlong $\mathrm{Yu}^{\mathrm{a}, *}$, Yanwei Pang ${ }^{\mathrm{a}}$, Jungong Han ${ }^{\mathrm{b}}$ \\ ${ }^{a}$ School of Electrical and Information Engineering, Tianjin University, China \\ ${ }^{b}$ School of Computing and Communications, Lancaster University, UK
}

\begin{abstract}
Zero-shot learning (ZSL) aims at recognizing unseen classes that are absent during the training stage. Unlike the existing approaches that learn a visual-semantic embedding model to bridge the low-level visual space and the high-level class prototype space, we propose a novel synthesized approach for addressing ZSL within a dictionary learning framework. Specifically, it learns both a dictionary matrix and a class-specific encoding matrix for each seen class to synthesize pseudo instances for unseen classes with auxiliary of seen class prototypes. This allows us to train the classifiers for the unseen classes with these pseudo instances. In this way, ZSL can be treated as a traditional classification task, which makes it applicable for traditional and generalized ZSL settings simultaneously. Extensive experimental results on four benchmark datasets (AwA, CUB, aPY, and SUN) demonstrate that our method yields competitive performances compared to state-of-the-art methods
\end{abstract}

Keywords: Zero-Shot Learning, Dictionary Learning, Image Recognition, Synthesized Model

\section{Introduction}

Deep learning greatly promotes the development of computer vision, such as object classification, image retrieval, and action classification. The performances of these tasks are usually evaluated after extensive and incremental training with a large amount of labeled data. However, real-world tasks only have a small quantity of or even no training data, giving rise to the failure of traditional classification models under such scenarios. Aiming to promote traditional classification models to recognize categories with few data, Zero-Shot Learning (ZSL) [1, 2, 3, 4, 5, 6] has attracted a lot of attention recently.

In ZSL, the training classes (seen classes) and test classes (unseen classes) are disjoint. Unseen object recognition is typically achieved by transferring knowledge from seen classes to unseen ones via a pre-defined class semantic space where both the seen and unseen classes are embedded. To this end, each class is associated with a vector in the class semantic space, which is called class prototype. Such a space can be structured with class attributes [7, 8, 9], or Word2Vec 10, 11. Specifically, attributes are obtained by manual annotation or automatic learning, while Word2Vec is obtained with language processing technology on a large text corpus.

The process of most current ZSL approaches generally consist of two steps: 1) learning the interaction relationships between the visual space and class prototype space;

\footnotetext{
*Corresponding authors

Email addresses: jizhong@tju.edu.cn (Zhong Ji), yuyunlong@tju.edu.cn (Yunlong Yu)
}

2) predicting the labels of test data with the semantic similarities between the test visual features and the unseen class prototypes using the learned model. Since the visual features and the class prototypes are located in different structural spaces, most existing approaches bridge the "heterogeneity gap" between visual and class prototype spaces using either a linear embedding model [12, 13], a bilinear embedding model [14, 15, 16, or a nonlinear model [17, 18].

These approaches can be indirectly compared with human being's inferential mechanism. In fact, ZSL can be addressed by imitating the human being's mechanism that recognizes a novel category. When finding a novel category, someone tends to classify it according to the consistence between the visual features and prior knowledge about the unseen classes. For example, as illustrated in Fig. 1, if a child has the prior knowledge of a horse and semantic description of "a unicorn is similar to a horse, apart from a unicorn has a long horn on the head", the child is very likely to accurately identify a unicorn the first time it is seen. The mechanism behind this is that the child can imagine a synthesized virtual appearance for a unicorn based on his/her prior knowledge.

Motivated by this observation, some recent works study ZSL in a more direct way, i.e., treating ZSL as a traditional classification task by synthesizing pseudo instances for unseen classes. Obviously, the key challenge lies in how to synthesize pseudo instances with prior knowledge about the seen classes and unseen ones. For example, [19] formulates it in a manifold learning framework, and [6, 20] present a diffusion regularization to ensure the balancing distribution of the synthesized data. 


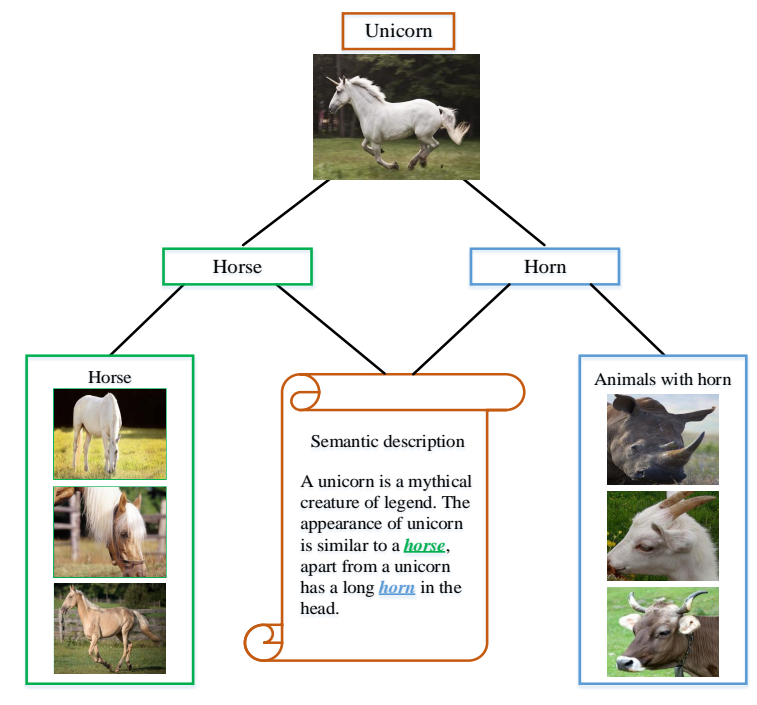

Figure 1: The mechanism behind human being that recognizes a novel category. Human can identify a unicorn the first time it is seen based on the prior knowledge of a horse and semantic description of "a unicorn is similar to a horse, apart from a unicorn has a long horn in the head".

In this paper, we also propose a synthesis approach to treat ZSL as a traditional classification task. Based on dictionary learning framework, reconstruction of visual features can be realized with the auxiliary of class prototypes sparsely, and the class-specific properties are preserved. Different with the existing synthesis approaches [6, 19, 20] that synthesize unseen instances with class prototypes, our approach synthesizes the pseudo unseen instances with not only their corresponding class prototypes but also their affinity seen classes.

Our approach is referred as Class-Specific Synthesized Dictionary Model (CSSD), which consisits of two stages: 1) Pseudo instances synthesis stage. In this stage, the proposed model undergoes two steps. First, it maps the seen class prototypes into a latent space to learn a class-specific encoding matrix for each class, and learns a dictionary matrix simultaneously to reconstruct the visual features within a dictionary learning framework. And then, it synthesizes pseudo instances of unseen classes with the class prototypes of affinity seen classes and their corresponding encoding matrices, in which the affinity seen classes represent those seen classes similar to unseen ones. 2) Prediction stage. Classifiers for unseen classes are first trained with these pseudo instances in a supervised way. Afterwards, the labels of test data are predicted by these classifiers. The flowchart of CSSD approach is illustrated in Fig. 2.

In summary, the contributions of our proposed approach can be summarized into two-fold:

- We propose a novel synthesized ZSL approach by synthesizing pseudo instances of unseen classes with their corresponding class prototypes and their affinity seen classes.

- To effectively synthesize unseen pseudo instances, we learn the common properties of all classes as well as the specific properties of each class within a dictionary learning framework. Specifically, it learns both a dictionary matrix and a class-specific encoding matrix for each seen class, so as to synthesize pseudo instances for unseen classes with auxiliary of seen class prototypes. In this way, ZSL is transferred to a traditional classification task.

\section{Related Work}

\subsection{Traditional Zero-Shot Learning}

The goal of ZSL is to recognize instances from unseen classes. And it is achieved by transferring knowledge from seen classes to unseen ones with a kind of class prototype space. The existing ZSL approaches can be divided into two different strategies, embedding-based ZSL and synthesisbased ZSL.

\subsubsection{Embedding-Based ZSL}

Embedding-based approaches treat ZSL as a multimodal learning problem that learns the interactions between the visual space and the class prototype space. They are divided into three sub-categories in terms of the direction of the embedding function. The first one [12, 17] learns an embedding function to project the visual features to the class prototype space. With the learned function, the test instance can obtain its class embedding vector in the class prototype space. By measuring the semantic similarities between the class embedding vector and unseen class prototypes, the test instance is predicted with the nearest neighbor classifier. However, these methods usually suffer from hubness issue 21. That is, a small number of objects (hubs) may occur as the nearest neighbour of many categories, resulting the diminishing of nearest neighbour method. In order to alleviate this issue, in the approaches of the second sub-category, [13]22 apply an opposite direction to map the class prototypes into the visual space. The last sub-category [23, 24, 25] learns a bilinear embedding function to project both the visual features and class prototypes into a shared latent space, which can preserve the compatibility scores between different modalities. The correct class usually has a higher score than other classes. Among these, JEDM 24] is the most related work to ours. The difference between JEDM and CSSD is that our model learns class-specific encoding matrices in terms of different classes while JEDM learns a common encoding matrix for all classes.

\subsubsection{Synthesis-Based ZSL}

Synthesis-based approaches treat ZSL as a traditional classification task by synthesizing pseudo instances with the unseen class prototypes. The existing approaches differ in the synthesis process. For example, [19] learns manifold 


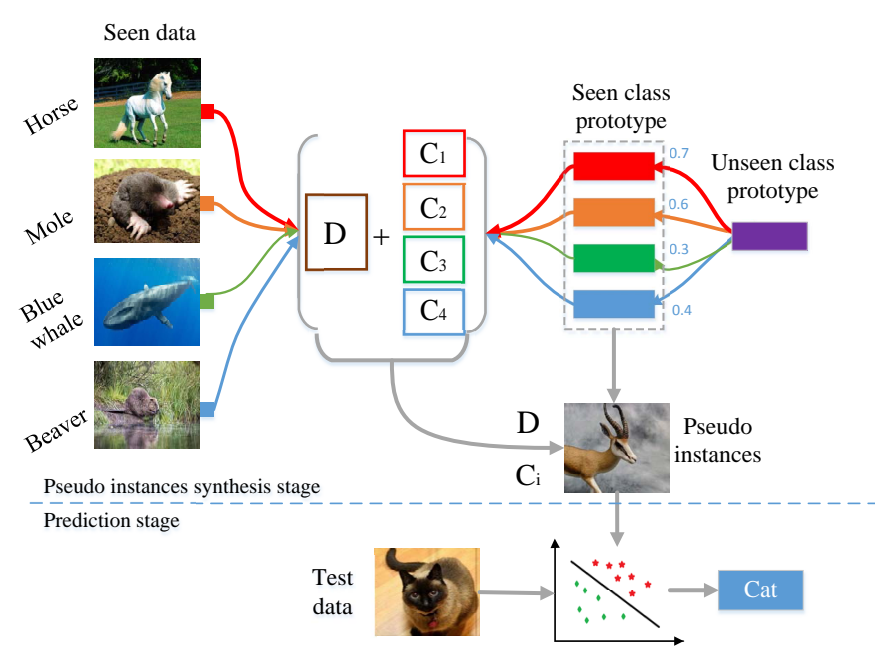

Figure 2: The illustration of our proposed CSSD model for ZSL. In the pseudo instances synthesis stage, it first learns a dictionary matrix D and a class-specific encoding matrix $\mathrm{C}_{i}$ for each seen class within a dictionary learning framework. Then, pseudo instances for unseen class are synthesized with the help of its affinity seen classes together with $\mathrm{D}$ and $\mathrm{C}_{i}$. In the prediction stage, classifiers for unseen classes are first trained with these pseudo instances in a supervised way. And then, the labels of test data are predicted by these classifiers.

structure in the class prototype space based on sparse coding and then transfers the manifold information into the visual space to synthesize pseudo instances for predicting the labels of unseen classes. [6, 20, present a diffusion regularization to ensure the balancing distribution of the synthesized data. The proposed CSSD is also a synthesis approach. Different with the existing synthesis approaches that synthesize pseudo unseen instances only with their corresponding class prototypes, the proposed CSSD synthesizes the pseudo instances of unseen classes with both their class prototypes as well as their affinity seen classes.

\subsection{Generalized Zero-Shot Learning}

The early ZSL work has a limitation that the learned model only can differentiate categories between unseen classes, which violates the reality. Recently, [17, 26] extend ZSL to a more general scene called Generalized Zero-Shot Learning (GZSL), where the test instances are classified into both the seen and unseen classes. [17] designs two novelty detection strategies to differentiate unseen classes from seen classes to help the final object classification. Since no training data are available for unseen classes, the learned model tends to classify the test instances into the seen classes. To alleviate this issue, [26] introduces a calibration factor to calibrate the classifiers for both seen and unseen classes. And [27] uses a maximum margin framework for semantic manifold-based recognition, which constrains the distance of vocabulary atoms to ensure the labeled images can be projected closest to their class prototypes than other classes.
Table 1: The notations used in CSSD model.

\begin{tabular}{|c|c|}
\hline Notations & Description \\
\hline$M$ & number of seen classes \\
\hline$N$ & number of unseen classes \\
\hline$m_{i}$ & number of seen instances of $i$-th class \\
\hline$n$ & number of unseen instances \\
\hline$p$ & dimensionality of visual features \\
\hline$q$ & dimensionality of class prototypes \\
\hline $\mathrm{X}_{i} \in \mathbb{R}^{p \times m_{i}}$ & visual features of each seen class \\
\hline $\mathrm{X}_{u} \in \mathbb{R}^{p \times n}$ & visual features of unseen classes \\
\hline $\mathrm{A}_{i} \in \mathbb{R}^{q \times m_{i}}$ & class prototypes of each seen class \\
\hline $\mathrm{A}_{u} \in \mathbb{R}^{q \times N}$ & class prototypes of unseen classes \\
\hline $\mathrm{D}$ & dictionary matrix \\
\hline $\mathrm{C}_{i}$ & class-specific encoding matrix of $i$-th class \\
\hline $\mathrm{P}_{i}$ & class-specific embedding function of $i$-th class \\
\hline Q & embedding function of all seen classes \\
\hline$a_{i}$ & class prototype of $i$-th seen class \\
\hline$a_{j}$ & class prototype of $j$-th unseen class \\
\hline$\mu_{i j}$ & class similarities of different classes \\
\hline$x_{j}^{p s e}$ & pseudo instances of $j$-th unseen class \\
\hline $\mathrm{A}_{j}^{p s e}$ & synthesized class prototypes \\
\hline $\mathrm{P}_{i}^{p s e}$ & embedding function of selected class \\
\hline
\end{tabular}

\section{The Proposed Model}

\subsection{Notations}

Suppose that we have $M$ seen classes in the training stage and $N$ unseen classes in the testing stage, and each class is associated with a $q$-dimensional class prototype vector in the class prototype space. We denote $\mathrm{X}=\left[\mathrm{X}_{1}, \mathrm{X}_{2}, \cdots, \mathrm{X}_{M}\right]$ as a set of $p$-dimensional visual features from $M$ seen classes, where $\mathrm{X}_{i} \in \mathbb{R}^{p \times m_{i}}$ is the visual feature set of class $i$, and $m_{i}$ is the number of seen instances of each class. Similarly, $\mathrm{A}_{i} \in \mathbb{R}^{q \times m_{i}}$ is denoted as the class prototypes of class $i$. Let $\left\{\mathrm{X}_{u}, \mathrm{~A}_{u}\right\}$ denote all the test data, in which $\mathrm{X}_{u} \in \mathbb{R}^{p \times n}$ is available only when predicting the labels. The notations used in this paper are summarized in Table 1.

\subsection{Reconstruction of Class-Specific Dictionary Learning Model}

The traditional dictionary learning aims at learning a dictionary matrix $\mathrm{D}$ to sparsely represent the input data $\mathrm{X}$ with its corresponding encoding matrix $\mathrm{C}$. The process is usually followed by an $l_{0}$ or $l_{1}$ norm constraint on the encoding matrix $\mathrm{C}$ to make it sparse. The model can be summarized as follows:

$$
\left\{\mathrm{D}^{*}, \mathrm{C}^{*}\right\}=\arg \min _{\mathrm{D}, \mathrm{C}}\|\mathrm{X}-\mathrm{DC}\|_{F}^{2}+\lambda\|\mathrm{C}\|_{p}+\psi(\mathrm{D}, \mathrm{C}, \mathrm{X})
$$

where $\lambda$ is a hyper-parameter, and $\psi(\mathrm{D}, \mathrm{C}, \mathrm{X})$ stands for discriminative functions to ensure the discrimination of $\mathrm{D}$ and $\mathrm{C}$.

Clearly, traditional dictionary models cannot achieve ZSL directly since they are learned only with visual features of seen classes. In order to achieve the knowledge 
transfer across different classes, we propose to embed the class prototypes to the dictionary learning model to reveal the relationships between the seen classes and unseen ones.

Due to the fact that redundant information exists in the class prototype space, denoting the class prototypes as the encoding matrix $\mathrm{C}$ directly will not ensure the sparsity of C. To address this problem, we propose to use an embedding function $\mathrm{P}$ to map the class prototypes into a latent space, which preserves the semantic relationships between different classes. And the redundant information of the seen class prototypes is decreased when we consider the mapped class prototypes as the encoding matrix. Inspired by this idea, we thus have:

$$
\min _{\mathrm{P}, \mathrm{C}}\|\mathrm{PA}-\mathrm{C}\|_{F}^{2}
$$

where the mapped class prototypes are considered as the encoding matrix $\mathrm{C}$. In this way, there is an advantage that the $l_{0}$ or $l_{1}$ norm constraint on the encoding matrix $\mathrm{C}$ can be removed, and the class prototypes can be embedded into the dictionary-based approach easily to achieve the purpose of knowledge transfer. Therefore, when integrating Eq. (2) into the dictionary learning framework, we have an objective function as follows:

$$
\begin{aligned}
\left\{\mathrm{P}^{*}, \mathrm{D}^{*}, \mathrm{C}^{*}\right\} & =\arg \min _{\mathrm{P}, \mathrm{D}, \mathrm{C}}\|\mathrm{X}-\mathrm{DC}\|_{F}^{2}+\|\mathrm{PA}-\mathrm{C}\|_{F}^{2} \\
& +\psi(\mathrm{D}, \mathrm{P}, \mathrm{C}, \mathrm{X}, \mathrm{A})
\end{aligned}
$$

The first term in Eq. (3) is used to jointly reconstruct the visual features of seen classes with the linear combination between the dictionary matrix $\mathrm{D}$ and the mapped class prototypes $\mathrm{C}$ in the latent space. The second term is to map class prototypes into a latent space of seen classes and unseen classes to realize knowledge transfer. And the last term $\psi(\mathrm{D}, \mathrm{P}, \mathrm{C}, \mathrm{X}, \mathrm{A})$ is a discriminative term to achieve other constraints.

However, due to the different distribution of each class, a global linear model is oversimple to represent complicated relationships between visual features and class prototypes of all seen classes. And a nonlinear model is easily overfitting on the seen classes. Thus, we prefer to learn a unique linear model for each class to better reflect their relationships. Based on this idea, instead of learning a common encoding matrix, we propose to learn a class-specific encoding matrix for each seenclass to preserve discriminative information. Thus, the objective function becomes:

$$
\begin{aligned}
\min _{\mathrm{D}, \mathrm{C}_{i}, \mathrm{P}_{i}, \mathrm{Q}} & \sum_{i=1}^{M}\left\|\mathrm{X}_{i}-\mathrm{DC}_{i}\right\|_{F}^{2}+\left\|\mathrm{P}_{i} \mathrm{~A}_{i}-\mathrm{C}_{i}\right\|_{F}^{2} \\
& +\lambda\left\|\mathrm{QA}_{i}-\mathrm{C}_{i}\right\|_{F}^{2}+\gamma\left\|\mathrm{P}_{i}\right\|_{F}^{2}+\|\mathrm{Q}\|_{F}^{2},\left\|d_{i}\right\|_{2}^{2} \leq 1
\end{aligned}
$$

The first term in Eq. (4) represents the reconstruction error in terms of different classes. $\mathrm{D}$ is the dictionary matrix shared by all seen classes, $\mathrm{C}_{i}$ and $\mathrm{X}_{i}$ represent the class-specific encoding matrix and visual features of each seen class, respectively. The second and third terms are the process of mapping class prototypes into a latent space, and the difference between them locates that the former distinguishes the discriminative information between different classes and the latter preserves the same parts. That is, $\mathrm{P}_{i}(1 \leq i \leq M)$ is thought to be the class-specific embedding function of each seen class, and Q is considered to be an embedding function shared by all different seen classes. And $\lambda$ is a hyper-parameter to trade off the proportion between them, which is determined through a cross-validation strategy. The last two terms are regularizers, where $\gamma$ is another hyper-parameter. Besides, $d_{i}$ denotes the $i$-th atom of the dictionary matrix D. Utilizing $l_{2}$ norm to constrain the value of the atom of dictionary matrix $\mathrm{D}$ is to make their distribution more balanced so as to make sure the model more stable.

Next, we introduce the optimization approach. When solving $\mathrm{D}, \mathrm{C}_{i}, \mathrm{P}_{i}$ and $\mathrm{Q}$ simultaneously, Eq. (4) is not a convex objective function while it is convex for solving each variable separately. The optimization can be done according to the following steps.

(1) Fix D, $\mathrm{P}_{i}$ and $\mathrm{Q}$, and update $\mathrm{C}_{i}$

$$
\begin{aligned}
\mathrm{C}_{i}^{*} & =\arg \min _{\mathrm{C}_{i}} \sum_{i=1}^{M}\left\|\mathrm{X}_{i}-\mathrm{DC}_{i}\right\|_{F}^{2}+\left\|\mathrm{P}_{i} \mathrm{~A}_{i}-\mathrm{C}_{i}\right\|_{F}^{2} \\
& +\lambda\left\|\mathrm{QA}_{i}-\mathrm{C}_{i}\right\|_{F}^{2}
\end{aligned}
$$

This is a standard least squares problem which can get its closed-form solution when we take the derivative of Eq. (5) with respect to $\mathrm{C}_{i}$ and make it equal to zero:

$$
\mathrm{C}_{i}^{*}=\left(\mathrm{D}^{T} \mathrm{D}+(1+\lambda) \mathrm{I}\right)^{-1}\left(\mathrm{DX}_{i}+\left(\mathrm{P}_{i}+\lambda \mathrm{Q}\right) \mathrm{A}_{i}\right)
$$

(2) Fix D and $\mathrm{C}_{i}$, update $\mathrm{P}_{i}$ and $\mathrm{Q}$

$$
\left\{\begin{array}{c}
\mathrm{P}_{i}^{*}=\arg \min _{\mathrm{P}_{i}} \sum_{i=1}^{M}\left\|\mathrm{P}_{i} \mathrm{~A}_{i}-\mathrm{C}_{i}\right\|_{F}^{2}+\gamma\left\|\mathrm{P}_{i}\right\|_{F}^{2} \\
\mathrm{Q}^{*}=\arg \min _{\mathrm{Q}} \sum_{i=1}^{M} \lambda\left\|\mathrm{QA}_{i}-\mathrm{C}_{i}\right\|_{F}^{2}+\|\mathrm{Q}\|_{F}^{2}
\end{array}\right.
$$

Due to $\mathrm{P}_{i}$ and $\mathrm{Q}$ are independent, we can obtain their closed-form solutions respectively, which are as follows:

$$
\left\{\begin{aligned}
\mathrm{P}_{i}^{*} & =\left(\mathrm{C}_{i} \mathrm{~A}_{i}^{T}\right)\left(\mathrm{A}_{i} \mathrm{~A}_{i}^{T}+\gamma \mathrm{I}\right)^{-1} \\
\mathrm{Q}^{*} & =\left(\sum_{i=1}^{M} \lambda \mathrm{C}_{i} \mathrm{~A}_{i}^{T}\right)\left(\sum_{i=1}^{M} \lambda \mathrm{A}_{i} \mathrm{~A}_{i}^{T}+\mathrm{I}\right)^{-1}
\end{aligned}\right.
$$

(3) Fix $\mathrm{C}_{i}, \mathrm{P}_{i}$ and $\mathrm{Q}$, update $\mathrm{D}$

$$
\mathrm{D}^{*}=\arg \min _{\mathrm{D}} \sum_{i=1}^{M}\left\|\mathrm{X}_{i}-\mathrm{DC}_{i}\right\|_{F}^{2},\left\|d_{i}\right\|_{2}^{2} \leq 1
$$

The optimization of $\mathrm{D}$ can be achieved by introducing an intermediate variable $\mathrm{S}$ :

$$
\mathrm{D}^{*}=\arg \min _{\mathrm{D}, \mathrm{S}} \sum_{i=1}^{M}\left\|\mathrm{X}_{i}-\mathrm{DC}_{i}\right\|_{F}^{2}, \text { s.t. } \mathrm{D}=\mathrm{S},\left\|s_{i}\right\|_{2}^{2} \leq 1
$$




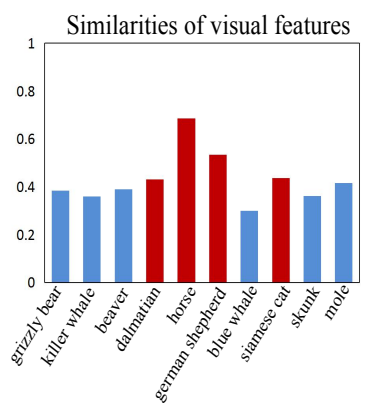

(a)

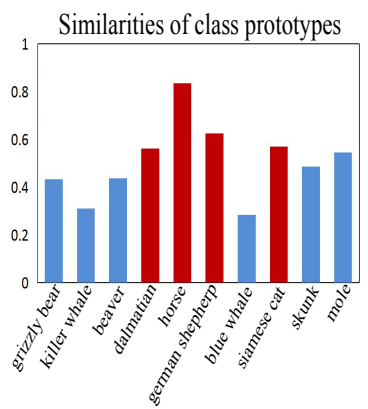

(b)
Figure 3: The consistency of similarities between visual features and class prototypes. In both sub-figures, the height of each column is the similarity between each class and "antelope". And the red columns correspond to affinity classes of "antelope".

The solution to Eq. (10) can be obtained by ADMM algorithm. When the difference between two adjacent iterations is less than a threshold, the optimization process stops.

\subsection{Synthesis of Pseudo Instances}

Most existing approaches directly utilize the model learned from seen classes to predict the labels of unseen classes 28, 29, 30. However, since the distribution of seen classes and unseen ones are different, the model learned from seen classes cannot be well generalized to unseen ones. To diminish this distribution gap, we propose to use the combination of affinity seen classes to replace unseen classes to train classifiers. We have the following assumption.

Assumption The semantic similarities among classes obtained with visual features are consistent with those obtained with class prototypes. Thus, the combination of affinity seen classes prototypes with the embedding model can approximate the visual features of unseen classes.

To verify the correctness of our assumption, we perform statistical studies on AwA dataset. Take the class "antelope" for example. As illustrated in Fig. 3, we can find that the similarities between the class "antelope" and the other ten classes with visual prototypes (i.e., the mean visual features of each class) are similar with those obtained with the class prototypes (i.e., class attributes). Specifically, "horse", "german shepherd", "siamese cat" and "dalmatian" are more similar than the others, such as "blue whale" in visual space (i.e., Fig.3 (a)). And this closeness are kept in the class prototype space (i.e., Fig.3 (b)). Thus, it verifies the consistency of similarities between visual features and class prototypes.

Based on this assumption, the pseudo instances of unseen classes can be synthesized with their most affinity seen classes. Therefore, the $j$-th pseudo instances $x_{j}^{p s e}$ can be synthesized with the following form:

$$
x_{j}^{p s e}=\sum_{i=1}^{k} \mu_{i j} \mathrm{D}\left(\mathrm{P}_{i}^{s e l}+\lambda \mathrm{Q}\right) a_{j},(1 \leq j \leq N)
$$

where $k$ denotes the number of selected affinity seen classes, $\mathrm{P}_{i}^{\text {sel }}$ is the embedding function corresponding to the $i$-th selected class, and $\mu_{i j}$ is the class similarity that is considered as the weight of each affinity class.

There are many possible ways to evaluate the similarities between different classes, such as cosine distance, Euclidean distance, and so on. In this work, we use cosine distance to measure the similarities between different classes

$$
\mu_{i j}=\frac{\left\langle a_{i}, a_{j}\right\rangle}{\left\|a_{i}\right\|_{2}\left\|a_{j}\right\|_{2}}
$$

where $a_{i}$ and $a_{j}$ are the class prototypes of the $i$-th seen class and the $j$-th unseen class.

We utilize SVM as the classifiers. Since enough labeled data are required to train SVM classifiers, we have to prepare several class prototypes for each unseen class in advance to synthesize enough pseudo instances.

In the visual space, data from different classes are separated and form a tight cluster. Thus we assume each class follows a Gaussian distribution. Considering the similarities among different classes in visual space are consistent with those in class prototype space, the prepared class prototypes $\mathrm{A}_{j}^{\text {pse }}$ should also follow a Gaussian distribution

$$
\mathrm{A}_{j}^{p s e}=a_{j}+\delta \cdot \mathrm{I}
$$

where $a_{j}$ serves as the mean value of Gaussian distribution and $\delta$ is the variance. Therefore, we are able to synthesize plenty of unseen class prototypes for the same class, which ensures the diversity of synthesized pseudo instances.

Algorithm 1 summarizes the process of our CSSD model.

\section{Experiments}

\subsection{Datasets and Settings}

In this section, we conduct extensive experiments on four benchmark datasets to illustrate the effectiveness and superiority of our proposed model. The four datasets are Animals with Attributes (AwA) 18, Caltech-UCSD Bird2011 (CUB) [31, aPascal-aYahoo (aPY) [7], and SUN Attribute (SUN) 32]. Table 2 summarizes the details of the four datasets.

Visual features. To compare fairly with the existing approaches, we use the VGG-19 [33] deep features extracted from popular CNN architecture as the visual features.

Class prototypes. In this paper, we choose both attributes and Word2Vec as the class prototypes for AwA and CUB datasets, respectively. The attributes are predefined and Word2Vec are obtained by a large text corpus based on a neural language processing technology. For aPY and SUN datasets, we only use attributes as the class prototypes since few competitors are evaluated with Word2Vec on them.

Implementation details. In our proposed model, there are four parameters $\lambda, \gamma, \sigma$, and $k$ need to be adjusted, in which $\lambda$ and $\gamma$ are two hyper-parameters in the 
Algorithm 1: The process of CSSD

Input:

1: The seen domain:

Visual features of each seen class $\mathrm{X}_{i} \in \mathbb{R}^{p \times m_{i}}$;

Class prototypes of each seen class $\mathrm{A}_{i} \in \mathbb{R}^{q \times m_{i}}$

Hyper-parameter $\lambda, \gamma$;

2: The unseen domain:

Visual features of unseen classes $\mathrm{X}_{u} \in \mathbb{R}^{p \times n}$;

Class prototypes of unseen classes $\mathrm{A}_{u} \in \mathbb{R}^{q \times N}$;

Parameter $\sigma, k$;

Output: The predicted labels of test data.

Training:

3: Repeat;

4: Update $\mathrm{C}_{i}$ according to Eq. (6);

5: Update $\mathrm{P}_{i}$ and $\mathrm{Q}$ according to Eq. (8);

6: Update D according to Eq. (10);

7: Until the iteration stops;

8: Return $\mathrm{P}_{i}, \mathrm{Q}$ and $\mathrm{D}$;

Synthesizing data:

9: Synthesize class prototypes of each unseen classes $\mathrm{A}_{j}^{\text {pse }}$ according to Eq. (13);

10: Compute class similarities $\mu_{i j}$ according to Eq. (12);

11: Synthesize pseudo instances $x_{j}^{\text {pse }}$ with affinity seen classes according to Eq. (11);

Testing:

12: Train SVM classifiers with the pseudo instances $x_{j}^{p s e}$;

13: Predict the labels of test data with the trained SVM classifiers.

Table 2: The statistics of the four datasets used in the experiments. '/' in columns of 'Instances' and 'Classes' represents the split of training/test instances and seen/unseen classes, respectively.

\begin{tabular}{|c|c|c|c|}
\hline Dataset & Instances & Attribute & Classes \\
\hline AwA & $24,295 / 6,180$ & 85 & $40 / 10$ \\
CUB & $8,855 / 2,933$ & 312 & $150 / 50$ \\
aPY & $12,695 / 2,644$ & 64 & $20 / 12$ \\
SUN & $14,140 / 200$ & 102 & $707 / 10$ \\
\hline
\end{tabular}

training stage, and $\sigma$ serves as the variance of the Gaussian distribution, and $k$ is the number of selected affinity seen classes. We use 5-fold cross-validation strategy to select the parameters with the best performance. That is, we split the training data into five parts, one for validation and the rest as the training set. Once the parameters are fixed, all seen instances form the training set to get the final model.

\subsection{Comparative Results of Traditional ZSL}

The performance of Traditional ZSL mainly indicates the transferability from seen classes to unseen ones of the ZSL approaches. In this part, we conduct Traditional ZSL experiments with both the attributes and Word2Vec.

First, we select seven attribute-based approaches for comparison, including 1) Convex Combination of Semantic Embedding (ConSE) [12], 2) Matrix tri-Factorization with
Table 3: Traditional ZSL results (\%) of different approaches on four datasets with attributes. For each column, the best one is marked in bold and the second best one is marked with underline.

\begin{tabular}{|c|c|c|c|c|}
\hline Method & AwA & CUB & aPY & SUN \\
\hline ConSE [12] & 64.3 & 33.6 & 34.5 & 74.5 \\
MFMR [34] & 79.8 & 47.7 & $\underline{48.2}$ & $\mathbf{8 4 . 0}$ \\
ESZSL [23] & 75.9 & 45.7 & 30.3 & 82.0 \\
JEDM [24] & 78.7 & 47.8 & 43.7 & 80.5 \\
SynC [22] & 74.9 & $\mathbf{5 3 . 7}$ & 27.9 & $\underline{83.0}$ \\
\hline RKT [19] & 73.3 & 40.2 & 44.3 & 82.0 \\
UVDS [20] & $\mathbf{8 2 . 1}$ & 44.9 & 42.3 & 80.5 \\
\hline CSSD (Ours) & $\underline{81.2}$ & $\underline{52.5}$ & $\mathbf{5 4 . 1}$ & $\underline{83.0}$ \\
\hline
\end{tabular}

Table 4: Traditional ZSL results (\%) of different approaches on AwA and CUB datasets with Word2Vec. For each column, the best one is marked in bold and the second best one is marked with underline.

\begin{tabular}{|c|c|c|}
\hline Method & AwA & CUB \\
\hline ConSE [12] & 50.5 & 30.7 \\
ESZSL [23] & 67.5 & 30.6 \\
JEDM [24] & 72.4 & 31.2 \\
SynC [22] & 67.3 & 30.9 \\
\hline RKT [19] & $\mathbf{7 6 . 9}$ & 28.9 \\
UVDS [20] & 62.9 & $\underline{32.1}$ \\
\hline CSSD (Ours) & $\underline{72.7}$ & $\mathbf{3 2 . 9}$ \\
\hline
\end{tabular}

Manifold Regularizations (MFMR) [34, 3) Embarrassingly Simple Approach to Zero-Shot Learning (ESZSL) [23, 4) Joint Embedding Dictionary Model (JEDM) [24], 5) Synthesized Classifiers for Zero-Shot Learning (SynC) [22], 6) Relational Knowledge Transfer for Zero-Shot Learning (RKT) [19, and 7) Zero-shot Learning Using Synthesised Unseen Visual Data with Diffusion Regularisation (UVDS) [20]. Specifically, the first five approaches are embeddingbased approaches, and the last two are synthesis-based approaches.

The comparison results are shown in Table 3. The results of MFMR [34] and UVDS [20] are cited directly from their published papers and the performances of the rest competitors are obtained with the released codes using the same features as our CSSD. We report their best performances after tuning parameters. The parameters are selected from $\{0.001,0.01,0.1,1,10,100,1000\}$.

As illustrated in Table 3, we observe that the proposed CSSD achieves state-of-the-art performances. Specifically, except the MFMR 34 and SynC 22, our CSSD is better than the other embedding-based approaches, indicating that CSSD learns a more effective embedding function by learning common properties of all classes and specific properties of each class within a dictionary learning framework simultaneously. Unlike the other embedding-based approaches that learn an embeding function directly, SynC 22 builds a weighted graph with pseudo class prototypes in the class prototype space, and constructs synthesized classifiers in the visual space, achieving best result on the CUB dataset. Compared with synthesis-based ones, CSSD achieves best performances on three datasets except AwA, 
Table 5: Generalized ZSL results (\%) of different approaches. For each column, the best one is marked in bold and the second best one is marked with underline.

\begin{tabular}{|c|c|c|c|c|c|c|c|c|c|c|c|c|}
\hline \multirow{2}{*}{ Method } & \multicolumn{4}{|c|}{ AwA } & \multicolumn{4}{|c|}{ CUB } & \multicolumn{4}{|c|}{ aPY } \\
\hline & U-U & U-T & S-S & S-T & U-U & U-T & S-S & S-T & $\mathrm{U}-\mathrm{U}$ & U-T & S-S & S-T \\
\hline 12 & (64.3 & $\begin{array}{ll}10.1 \\
\end{array}$ & 81.6 & 81.1 & 33.6 & $\overline{5.1}$ & 54.8 & 53 & 34.5 & 3.1 & 71.7 & 71.2 \\
\hline MFMR 34 & $\underline{79.8}$ & 18.3 & 77.5 & 76.2 & 47.7 & 13.7 & 48.6 & 45.3 & 48.2 & 7.1 & 67.1 & 65.2 \\
\hline 23 & 75.9 & 24.1 & 83.4 & 82.2 & 45.7 & 17.7 & 63.1 & 60.8 & 27.9 & 17.3 & 77.3 & 73.2 \\
\hline JEDM [24] & 78.7 & 17.3 & 75.2 & 72.8 & 47.8 & 14.7 & 38.8 & 34.4 & 43.7 & 22.1 & 73.9 & 65.8 \\
\hline SynC 22 & 74.9 & 10.7 & $\underline{85.9}$ & 85.7 & 53.7 & 17.5 & 64.7 & 63.9 & 27.9 & 11.9 & $\underline{80.1}$ & 73.7 \\
\hline RKT [19] & 73.3 & 45.6 & 79.7 & 78.1 & 40.2 & 12.4 & 53.2 & 51.9 & 44.3 & $\underline{19.5}$ & 72.9 & 70.7 \\
\hline CSSD (Ours) & 81.2 & $\underline{34.7}$ & 87.5 & 87.1 & $\underline{51.2}$ & 19.1 & $\underline{63.2}$ & $\underline{62.7}$ & 54.1 & 8.7 & 84.9 & 84.2 \\
\hline
\end{tabular}

on which CSSD is only $0.9 \%$ lower than UVDS [20]. Since CSSD synthesizes pseudo instances with affinity seen classes and lacks the consideration of the balanced distribution on each dimension.

Next, we select Word2Vec as the class prototypes and conduct experiments on the same approaches except MFMR [34] since no performance is available in the original paper. The performances of different approaches are summarized in Table 4 . From the results, we can find that our model outperforms all the embedding-based approaches. Besides, CSSD beats UVDS 20] but is inferior than RKT [19] on AwA dataset. This is may be that different kinds of class prototypes have different effects.

\subsection{Comparative Results of Generalized ZSL}

Compared with Traditional ZSL, Generalized ZSL evaluates not only the transferability from seen classes to unseen ones but also the discriminability across both the seen and unseen classes. In this part, we conduct a set of experiments on AwA, CUB, and aPY datasets under the Generalized ZSL setting.

There are four evaluation scenarios under the Generalized ZSL setting, including U-U, U-T, S-S, and S-T. Specifically, U-U is the same as the setting of Traditional ZSL, which means classifying test data from unseen classes into the candidate unseen classes. U-T means classifying the test data from unseen classes into the joint space of seen classes and unseen ones. While S-S is the setting of multi-class classification actually, which means the test data are from seen classes and the candidate classes are the seen classes as well, and $\mathrm{S}-\mathrm{T}$ is a scenario that classifies the test data from seen classes into the joint space. For S-S, we randomly select $20 \%$ of the seen instances to be the test data, and the remaining seen instances are used to train the model. While for S-T, we also select $20 \%$ of the instances from seen classes and merge them with the instances from unseen classes to compose the test data.

We select six attribute-based approaches for comparison. Table 5 summarizes the best results of different models, the parameters of which are fine tuned by ourselves using the codes released in the original papers. From the results, we observe that the performances of S-T are close to those of S-S, indicating that most of the test data from seen classes are classified correctly. However, the performances of U-T decrease more obviously than those of U-U, indicating that CSSD is less discriminative to distinguish differences between seen classes and unseen ones. These observations illustrate that the Generalized ZSL is a more challenging task than Traditional ZSL. On AwA dataset, compared with embedding-based approaches, our model achieves best performances under four different scenarios, which obtains an improvement of $1.4 \%, 10.6 \%, 0.6 \%$ and $1.5 \%$ over the second best approach, respectively. On aPY dataset, we also achieve the best performances except for $\mathrm{U}-\mathrm{T}$. The reason locates that the aPY dataset is a coarsegrained dataset where classes share little information with each other, resulting that the class-specific encoding matrices learn ineffective discriminative information. For CUB dataset, the performances of our CSSD achieve the best performance on U-T, and the second-best performance on the other scenarios. While considering the discriminability of the pseudo instances, we can find the performances of CSSD are lower than RKT 19 on AwA and aPY datasets. This is because the synthesis process of CSSD preserves more transferable information other than discriminative information on coarse-grained datasets compared with RKT [19. Specifically, the discriminability of attributes on coarse-grained dataset is higher than that on fine-grained dataset. Compared with RKT [19] whichsynthesizes pseudo instances with all seen classes, CSSD synthesizes pseudo instances only with affinity seen classes and ignores dissimilar classes, and this method makes CSSD less discriminability than RKT.

\subsection{Parameter Sensitivity Analysis}

There are two hyper-parameters in the training stage, and a parameter in the process of synthesizing pseudo visual features. $\lambda$ is the hyper-parameter to trade-off the weight of the same parts and discriminative parts between different classes, and $\gamma$ is the balance parameter of regularizer. Besides, $k$ is the number of the selected affinity seen classes. To evaluate their influences, we conduct a list of experiments on AwA and CUB datasets. In the experiments, we change one parameter while fixing the others with their best values.

Fig. 4 illustrates the influences of different $\lambda$ on both datasets. We can find that the curves vary on different 
AwA

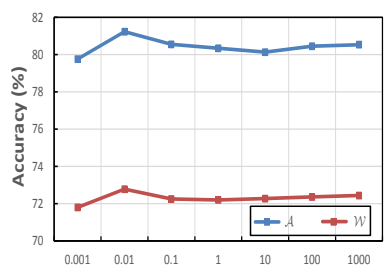

(a)
CUB

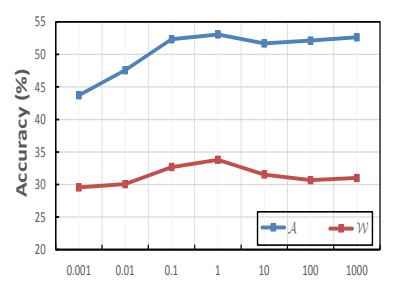

(b)
Figure 4: The influences of different $\lambda$ on AwA and CUB datasets with attributes and Word2Vec, respectively. And $\mathcal{A}$ and $\mathcal{W}$ are short for attributes and Word2Vec.

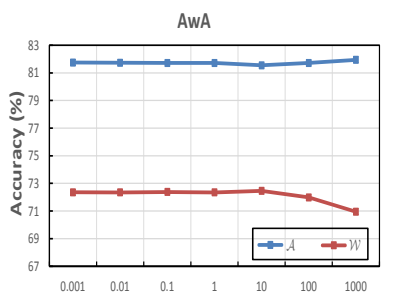

(a)

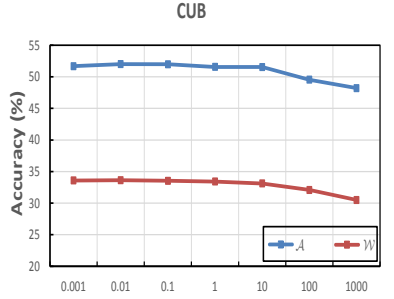

(b)
Figure 5: The influences of different $\gamma$ on AwA and CUB datasets with attributes and Word 2 Vec, respectively. And $\mathcal{A}$ and $\mathcal{W}$ are short for attributes and Word2Vec.

datasets. The curves in Fig.4 (a) show that the performances initially increase with the increase of $\lambda$ and achieve their peaks, and then decline on the AwA dataset. The performances achieve their best value when $\lambda$ is equal to 0.01 , illustrating that although the discriminative information plays a more important role than the same parts among different classes, preserving the information of same parts is also necessary. On CUB dataset, the overall trends are similar with Fig.4 (a), while the curses achieve their peaks when $\lambda$ is equal to 1 . We report the performances with their best value on the peak.

Two sub-figures in Fig. 5 show the effects of different $\gamma$ on both datasets. The performances are robust with the increase of $\gamma$ on the AwA dataset with both attributes and Word2Vec. When the value is larger than 10, the curves begin fluctuating slightly. On CUB dataset, the trends of curves are similar with that of AwA dataset, revealing the insensitivity of $\gamma$.

When evaluating the influence of parameter $k$, its range should be set in advance according to the number of seen classes. Specifically, we set it is $\{5,10,15,20,25,30\}$ on AwA dataset and $\{20,30,40,50,60,70\}$ on CUB dataset, respectively. Fig. 6 shows the trend of the accuracy curves with the increase of $k$ on both datasets. And we can observe that the two curves of $\mathcal{A}$ and $\mathcal{W}$ have the similar trend, i.e., rise or fall almost at the same number on both datasets. On AwA dataset, the accuracy reaches maximum when $k$ is equal to 15 , whereas on CUB dataset the number is 30 . The reason behind this phenomenon is that the

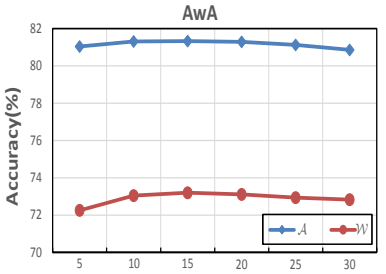

(a)

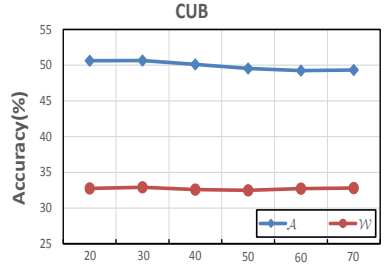

(b)
Figure 6: The influences of different $k$ on AwA and CUB datasets with attributes and Word2Vec, respectively. And $\mathcal{A}$ and $\mathcal{W}$ are short for attributes and Word2Vec.
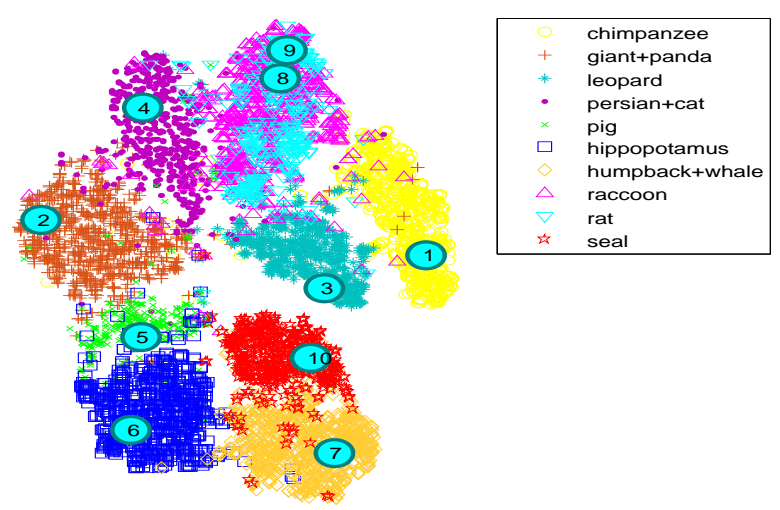

Figure 7: t-SNE visualization for our synthesized pseudo instances on AwA dataset.

\section{coarse-grained AwA dataset has fewer similar categories} than that of fine-grained CUB dataset.

\subsection{Further Analysis}

With the learned model, the pseudo instances of unseen classes are synthesized with class prototypes of their affinity seen classes and their corresponding class-specific encoding matrices. Given the unseen class prototype, we consider it as the mean value of the Gaussian distribution, while the variance is determined by 5 -fold cross-validation. We select the best value of $\sigma$ to visualize them with tSNE approach, as illustrated in Fig. 7. It can be observed that the pseudo instances from the same class are gathered around their corresponding class prototypes. Although each cluster of pseudo instances has a little overlap, the pseudo instances are enough to form separate clusters for the ten unseen classes, showing that the proposed CSSD approach can effectively reveal the visual distribution of the unseen classes.

\section{Conclusion}

In this paper, we proposed a novel approach for ZSL by synthesizing pseudo instances for unseen classes within a dictionary learning framework. It learns a dictionary matrix and a class-specific encoding matrix for each seen class to connect the interactions between the visual space 
and the class prototype space. The distribution of unseen classes is then synthesized with their affinity seen classes using the learned model. The experimental results on four benchmark datasets illustrate that the proposed CSSD approach achieves the state-of-the-art performances on both Traditional ZSL and Generalized ZSL tasks.

\section{Acknowledgments}

This work was supported by the National Natural Science Foundation of China under Grants 61771329, 61472273, and 61632018 .

\section{References}

[1] X. Li, M. Fang, J. Wu, Zero-shot classification by transferring knowledge and preserving data structure, Neurocomputing 238 (2017) 76-83.

[2] Y. Yu, Z. Ji, J. Guo, Y. Pang, Zero-shot learning with regularized cross-modality ranking, Neurocomputing 259 (2017) 14-20.

[3] M. Liu, D. Zhang, S. Chen, Attribute relation learning for zeroshot classification, Neurocomputing 139 (2014) 34-46.

[4] M. Elhoseiny, B. Saleh, A. Elgammal, Write a classifier: Zeroshot learning using purely textual descriptions, in: IEEE International Conference on Computer Vision, 2014, pp. 2584-2591.

[5] Y. Li, D. Wang, H. Hu, Y. Lin, Y. Zhuang, Zero-shot recognition using dual visual-semantic mapping paths, in: IEEE Conference on Computer Vision and Pattern Recognition, 2017, pp. 52075215.

[6] Y. Long, L. Liu, L. Shao, F. Shen, G. Ding, J. Han, From zeroshot learning to conventional supervised classification: Unseen visual data synthesis, in: IEEE Conference on Computer Vision and Pattern Recognition, 2017, pp. 6165-6174.

[7] A. Farhadi, I. Endres, D. Hoiem, D. Forsyth, Describing objects by their attributes, in: IEEE Conference on Computer Vision and Pattern Recognition, 2009, pp. 1778-1785.

[8] C. H. Lampert, H. Nickisch, S. Harmeling, Attribute-based classification for zero-shot visual object categorization, IEEE Transactions on Pattern Analysis and Machine Intelligence 36 (3) (2014) 453-465.

[9] S. J. Hwang, F. Sha, K. Grauman, Sharing features between objects and their attributes., in: IEEE Conference on Computer Vision and Pattern Recognition, 2011, pp. 1761-1768.

[10] T. Mikolov, I. Sutskever, K. Chen, G. Corrado, J. Dean, Distributed representations of words and phrases and their compositionality, Advances in Neural Information Processing Systems 26 (2013) 3111-3119.

[11] J. Pennington, R. Socher, C. Manning, Glove: Global vectors for word representation, in: Conference on Empirical Methods in Natural Language Processing, 2014, pp. 1532-1543.

[12] M. Norouzi, T. Mikolov, S. Bengio, Y. Singer, J. Shlens, A. Frome, G. S. Corrado, J. Dean, Zero-shot learning by convex combination of semantic embeddings, in: International Conference on Learning Representations, 2014, pp. 1-9.

[13] Y. Shigeto, I. Suzuki, K. Hara, M. Shimbo, Y. Matsumoto, Ridge regression, hubness, and zero-shot learning, in: The European Conference on Machine Learning and Knowledge Discovery in Databases, 2015, pp. 135-151.

[14] Z. Ji, Y. Xie, Y. Pang, L. Chen, Z. Zhang, Zero-shot learning with multi-battery factor analysis, Signal Processing 138 (2017) 265-272.

[15] A. Frome, G. S. Corrado, J. Shlens, S. Bengio, J. Dean, T. Mikolov, et al., Devise: A deep visual-semantic embedding model, in: Advances in Neural Information Processing Systems, 2013, pp. 2121-2129.

[16] Y. Fu, T. M. Hospedales, T. Xiang, Z. Fu, S. Gong, Transductive multi-view embedding for zero-shot recognition and anno- tation, in: European Conference on Computer Vision, 2014, pp. 584-599.

[17] R. Socher, M. Ganjoo, C. D. Manning, A. Ng, Zero-shot learning through cross-modal transfer, in: Advances in Neural Information Processing Systems, 2013, pp. 935-943.

[18] C. H. Lampert, H. Nickisch, S. Harmeling, Learning to detect unseen object classes by between-class attribute transfer, in: IEEE Conference on Computer Vision and Pattern Recognition, 2009, pp. 951-958.

[19] D. Wang, Y. Li, Y. Lin, Y. Zhuang, Relational knowledge transfer for zero-shot learning., in: AAAI, 2016, pp. 2145-2151.

[20] Y. Long, L. Liu, F. Shen, L. Shao, X. Li, Zero-shot learning using synthesised unseen visual data with diffusion regularisation, IEEE Transactions on Pattern Analysis and Machine Intelligence (2018) 1-14.

[21] A. Lazaridou, G. Dinu, M. Baroni, Hubness and pollution: Delving into cross-space mapping for zero-shot learning, in: Meeting of the Association for Computational Linguistics and the International Joint Conference on Natural Language Processing, 2015, pp. 270-280.

[22] S. Changpinyo, W. L. Chao, B. Gong, F. Sha, Synthesized classifiers for zero-shot learning, in: IEEE Conference on Computer Vision and Pattern Recognition, 2016, pp. 5327-5336.

[23] B. Romera-Paredes, P. H. S. Torr, An embarrassingly simple approach to zero-shot learning, in: International Conference on International Conference on Machine Learning, 2015, pp. 21522161.

[24] Y. Yu, Z. Ji, X. Li, J. Guo, Z. Zhang, H. Ling, F. Wu, Transductive zero-shot learning with a self-training dictionary approach, IEEE Transactions on Cybernetics (2018) 1-12.

[25] Z. Akata, S. Reed, D. Walter, H. Lee, B. Schiele, Evaluation of output embeddings for fine-grained image classification, in: IEEE Conference on Computer Vision and Pattern Recognition, 2015, pp. 2927-2936.

[26] W. L. Chao, S. Changpinyo, B. Gong, F. Sha, An empirical study and analysis of generalized zero-shot learning for object recognition in the wild, in: European Conference on Computer Vision, 2016, pp. 52-68.

[27] Y. Fu, L. Sigal, Semi-supervised vocabulary-informed learning, in: IEEE Conference on Computer Vision and Pattern Recognition, 2016, pp. 5337-5346.

[28] Z. Akata, F. Perronnin, Z. Harchaoui, C. Schmid, Labelembedding for attribute-based classification, in: IEEE Conference on Computer Vision and Pattern Recognition, 2013, pp. 819-826.

[29] Y. Xian, Z. Akata, G. Sharma, Q. Nguyen, M. Hein, B. Schiele, Latent embeddings for zero-shot classification, in: IEEE Conference on Computer Vision and Pattern Recognition, 2016, pp. 69-77.

[30] R. Qiao, L. Liu, C. Shen, A. V. D. Hengel, Less is more: zeroshot learning from online textual documents with noise suppression, in: IEEE Conference on Computer Vision and Pattern Recognition, 2016, pp. 2249-2257.

[31] C. Wah, S. Branson, P. Welinder, P. Perona, S. Belongie, The caltech-ucsd birds200-2011 dataset, California Institute of Technology.

[32] G. Patterson, C. Xu, H. Su, J. Hays, The sun attribute database: Beyond categories for deeper scene understanding, International Journal of Computer Vision 108 (1-2) (2014) 5981.

[33] K. Simonyan, A. Zisserman, Very deep convolutional networks for large-scale image recognition, in: International Conference on Learning Representations, 2014.

[34] X. Xu, F. Shen, Y. Yang, D. Zhang, H. T. Shen, J. Song, Matrix tri-factorization with manifold regularizations for zero-shot learning, in: IEEE Conference on Computer Vision and Pattern Recognition, 2017, pp. 2007-2016. 